\title{
IMPACT OF QUEUING ON CALL COMPLETION RATE IN GSM NETWORKS
}

\author{
M. A. Nkop' ${ }^{1}$ K. M. Udofia² and M. Umoren ${ }^{3}$ \\ 1,2,3 Dept of Electrical, Electronics and Computer EngineERING, Univ. of Uyo, Uyo, AKWA Ibom State. NigERIA \\ E-mail Addresses: ${ }^{1}$ keme4good@gmail.com, ${ }^{2}$ kmudofia@gmail.com, ${ }^{3}$ mfon4gigis@yahoo.com
}

\begin{abstract}
Effective utilization of network resource reduces the probability that a call arriving at the base station (BS) of a network will be lost. Performance evaluation plays an important role in modelling and designing of effective schemes to utilize limited network resource. This objective is achieved by an accurate traffic characterization and a precise analysis of the performance metrics in terms of traffic intensity. This has prompted the deployment of various concepts and techniques aimed at delivering solutions on the issue of optimization of GSM networks. In this paper, we use the queuing approach to develop a model for call completion, making signal power considerations as well. A General User Interface (GUI) is designed for the developed model using MATLAB and the impact of queuing on call completion is analysed by carrying out an assessment of the performance of the model at different parametric values.
\end{abstract}

Keywords: Base station, GSM networks, Network resource, Performance evaluation, Call completion, Queuing.

\section{INTRODUCTION}

Every network user who has satisfied the demands of network providers expects to complete every call made, but this is not always obtainable due to several performance issues $[3,13,19]$. High traffic intensity is known to have a profound negative effect on the performance of GSM networks; as such a system capacity commensurate with service high traffic demands $[2,4,17]$ is needed. Network capacity expansion may not always be cost effective, hence the need for the deployment of suitable techniques which can bring about an optimal use of limited network resources, while saving costs as well $[7,19]$.

The concept of queuing is very useful in modelling of systems where traffic management is of utmost importance. In GSM networks, traffic characteristics have a random behaviour in terms of arrival; usually following a Poisson's distribution $[1,6,10]$. The deployment of queuing in GSM traffic modelling can be very useful in effective channel utilization. The variations of queuing theory deployed in GSM modelling include prioritized and non-prioritized schemes $[6,17]$. The queue disciplines commonly used are the first in first out (FIFO); where customers are served on a first-in first-out basis, last in first out (LIFO); where customers are served in a last-in first- out manner, served in random order (SIRO); where customers are served in random order, and priority; where customers are served in order of their importance on the basis of their service requirements $[1,8,17][1]$. In this paper, we assess the impact of queuing on the performance of GSM networks at different traffic loads, as well the effect of queue size as traffic load increases.

\section{REVIEW OF RELATED WORKS}

Fang [1] did a performance evaluation of wireless cellular networks, where a realistic assumption approach was adopted in the analysis of call connection performance and mobility management where time variables such as call holding time, cell residence time, channel holding time, registration area residence time and inter-service time were all assumed to be generally distributed.

Ayeni [13] made a quantitative study of the phenomenon of call setup failure rate in Nigeria. Test calls were made to different identified routes of the network vis-à-vis intra, inter, Public Switched Telephone Network (PSTN) and international routes. It was discovered that relatively low call failure rates were recorded on intra and international routes as against inter and PSTN routes. It was also observed 
that definite call failure patterns could not be observed when viewed over the hours of the day, as is expected in normal traffic studies.

Efthymoglou, Pattaramalai, and Aalo, [14] derived simple closed-form expressions that closely approximate the call completion probability in a wireless cellular network under generalised gamma distributed cell dwell time, and call holding time with arbitrary distribution.

Ozawa,Takahashi and Takahashi, [15] proposed a method to obtain upper and lower bounds of call completion probabilities in a mobile communication network having a large number of base stations. To overcome the difficulty of analysing overlapping zones in the network, two smaller size models were introduced, and the upper and lower bounds were represented. It was seen that the values of the bounds could be gotten with less computation.

Mohammed M. Alwakeel, [10] investigated the impact of the type of the statistical distributions used to model channel holding time in cellular system on handoff failure probability and premature call termination probability. The analytical model investigated in his research showed that the type of the channel holding time distribution has no impact on the handoff failure probability and premature call termination probability. Instead, the mean of the channel holding time which is a constant value that depends on the parameters of the system such as the design layout and users distribution in the service area, regardless of the type of distribution used to model the channel holding time, has direct impact on the handoff failure probability and premature call termination probability.

Adegoke and Babalola [12] did an appraisal of the performance of GSM operators using Nigeria as a case study and examined the problems facing the industry. After evaluating the parameters that attributed to poor QoS by operators, they came up with suggestions towards improving network performance.

Osahenvenwem, Edeko and Emergbetere [4] proposed a scheme for traffic modelling that would predict blocking probability for voice call using channel fragmentation and pre-emptive prioritization. An analytical approach was deployed to determine a traffic model, assuming a certain number of channels in cell system, which was also homogenous in the entire mobile communication network. Queuing theory was deployed as a modelling technique, and calls were assumed to enter the system in an orderly sequence.

\section{METHODOLOGY}

The $M / M / 1$ queuing approach is adopted in this model $[4,17]$. We consider a system made up of many homogenous cells. These cells are assumed to be identical in capacity, performance and characteristics [5]. Only one cell will be modelled, being that the results are applicable to other cells. All traffic requests are treated as incoming calls in this analysis. Also, this is not a multiclass model in that only voice calls are considered.

\subsection{System Model:}

The basic assumptions to consider for the system model include the following;

i. A cell of size $\mathrm{C}_{\mathrm{n}}$ channels.

ii. A queue of infinite size.

iii. Signal power $\phi$, for incoming call must be at least $75 \%$, for acceptance of such a call.

iv. There is a timer set for each call in the queue; the call is lost once it exceeds the set time.

v. If a call finds no free channel on arrival, it is placed in the queue. If the call moves out of the cell before it is assigned a channel, it is deleted from the queue.

vi. The dwell time is exponentially distributed with mean $=1 / \mu$.

vii. The overall service time or call duration, $\mu=\mu_{C_{n}}+\mu_{Q}$

Where $\mu_{C_{n}}$ and $\mu_{Q}$ represent the channel holding time and queuing time respectively.

\subsection{Model Description}

Let us consider a base station with $\mathrm{C}_{\mathrm{n}}$ channels. In this scheme, no priority is given to any request (new call or handoff), all calls are seen as incoming calls. Therefore, all the channels are shared equally by both the new calls and handoff requests. The scheme also incorporates a queuing concept which is implemented using the first-in first-out protocol $[1,17]$. It is also assumed that the signal powers of both requests are good enough by a factor of $\phi$. In this scheme, both the signal strength and channel availability are considered. The arrival rate of incoming calls is $\lambda$, since any poor signal-quality request is dropped immediately, the effective incoming rate becomes $\phi \lambda$. Where $\phi$ represents the signal power factor.

Since this is a non-prioritized case, the effective call service time as earlier stated, is given as $\mu=\mu_{C_{n}}+\mu_{Q}$ 
, Where $\mu_{\mathrm{C}_{\mathrm{n}}}$ and $\mu_{\mathrm{Q}}$ are the channel holding time and queuing time respectively[19]. Figure 1 gives a detailed description of the proposed model.

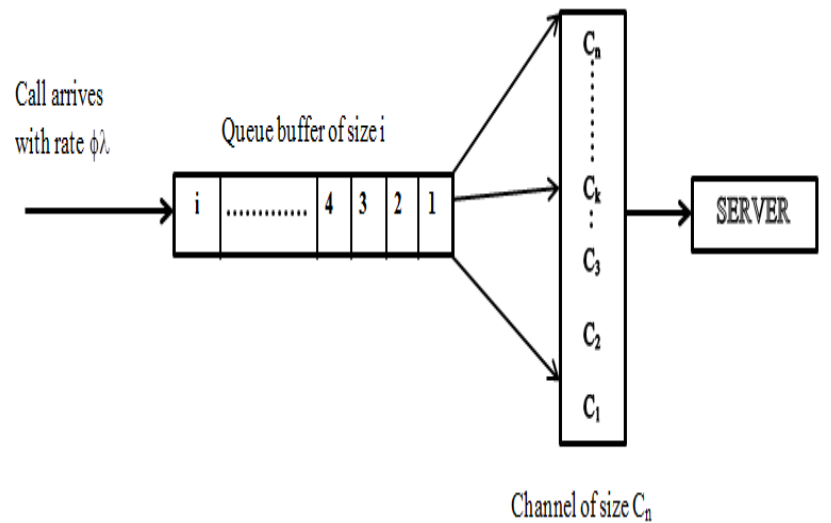

Figure 1: System model for proposed scheme.

In the base station (BS), there is a queue buffer of infinite size for all incoming calls (both new and handoff calls). On arrival, the signal power is assessed to ensure that it does not fall below the acceptable value. If all channels are busy, the incoming call is placed in a queue and served based on first in first out basis (F.I.F.0). The queue has a timer, and calls are cleared from the queue when the timer elapses. This is done to avoid a situation where there are too many requests waiting in the queue. Also, a call is cleared from the queue when the call moves out of the cell under consideration.

Applying the Markov one-dimensional process approach, $[9,14]$ the system model is illustrated in Figure 2. The state transition diagram for the proposed scheme as shown in Figure 2 is a Markov chain showing the various states of the system, from the point the call is put in the queue till when it accesses a channel.

We define the system state $i(i=0,1,2 \ldots ., \infty)$ of the cell as the sum of channels in the cell and the queue size. From the state transition diagram (Figure 2), the equilibrium probabilities can be derived as shown.

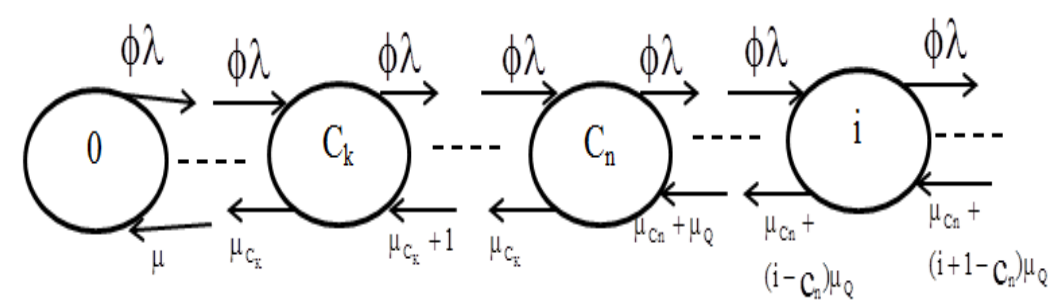

Figure 2: State Transition Diagram for System Model.

Applying the birth-death process $[1,4,10]$, the balance equations:

$$
\left\{\begin{array}{cc}
i \mu(i)=\phi \lambda P(i-1), & 0 \leq i \leq C_{n} \\
{\left[\mu_{C_{n}}+\left(i-c_{n}\right)\left(\mu_{C_{n}}+\mu_{C_{Q}}\right)\right] P(i)=\phi \lambda P(i-1),} & C_{n}<i \leq \infty
\end{array}\right.
$$

Using equation 1 recursively with the normalisation condition [1]

$$
\sum_{i=0}^{C_{n}} P(i)=1
$$

The steady state probability $\mathrm{P}(\mathrm{i})$ is derived as

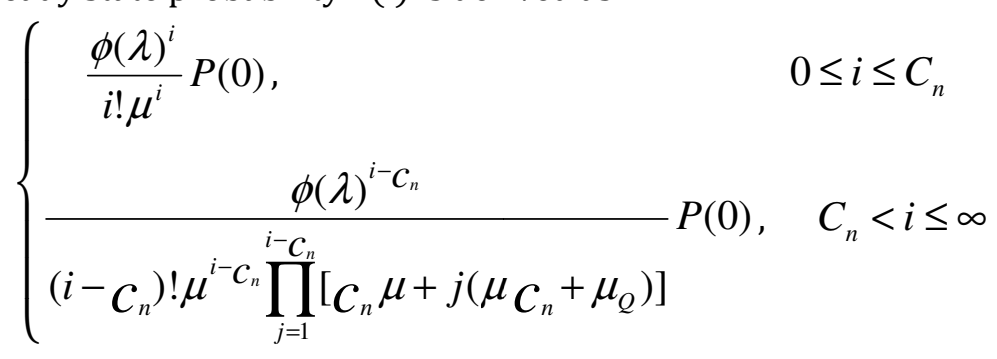

Simplifying, we have

$$
\begin{cases}\frac{1}{i} \phi\left(\frac{\lambda}{\mu}\right)^{i} P(0), & 0 \leq i \leq C_{n} \\ \frac{1}{\left(i-c_{n}\right) !} \times \phi\left(\frac{\lambda}{\mu}\right)^{i-c_{n}} \times \frac{1}{\prod_{j=1}^{i-c_{n}} c_{n} \mu+j\left(\mu_{C_{n}}+\mu_{Q}\right)} \times P(0), & C_{n}<i \leq \infty\end{cases}
$$


Where $P(0)$ can be given as

$$
P(0)=\left[\sum_{i=0}^{c_{n}} \frac{1}{i !} \phi\left(\frac{\lambda}{\mu}\right)^{i}+\sum_{i=c_{n}+1}^{\infty} \frac{1}{\left(i-c_{n}\right) !} \phi\left(\frac{\lambda}{\mu}\right)^{\left(i-c_{n}\right)} \times \frac{1}{\prod_{j=1}^{i-c_{n}}\left[c_{n} \mu+j\left(\mu_{C_{n}}+\mu_{Q}\right)\right]}\right]^{-1}
$$

The expression $\frac{\lambda}{\mu}$ is known as the traffic intensity [4][1] and is often represented as $\rho$

Therefore, $\rho=\frac{\lambda}{\mu}$ Substituting in equation 5 , it becomes

$$
P(0)=\left[\sum_{i=0}^{c_{n}} \frac{\phi \rho^{i}}{i !}+\sum_{i=c_{n}+1}^{\infty} \frac{\left.\phi \rho^{(i-} c_{n}\right)}{\left(i-c_{n}\right) !} \times \frac{1}{\prod_{j=1}^{i=c_{n}} c_{n} \mu+\left(\mu_{C_{n}}+\mu_{Q}\right)}\right]^{-1}
$$

Loss probability can be defined here as the probability that any call which accesses the network is not completed [1][3]. It can also be referred to as call failure probability. The loss probability is given as

$$
P_{L}=\sum_{i=0}^{\infty} P\left(c_{n}+i\right)
$$

Therefore,

$$
P_{L}=\left[\frac{\phi \rho^{\left(i-c_{n}\right)}}{\left(i-c_{n}\right) !} \times \frac{1}{\prod_{j=1}^{i-c_{n}} c_{n} \mu+j\left(\mu_{C_{n}}+j\left(\mu_{C_{n}}+\mu_{Q}\right)\right.}\right]\left[\sum_{i=0}^{c_{n}} \frac{\phi \rho^{j}}{i !}+\sum_{i=c_{n}+1}^{\infty} \frac{\left.\phi \rho^{(i-} c_{n}\right)}{\left(i-c_{n}\right) !} \times \frac{1}{\prod_{j=1}^{i=c_{n}} c_{n} \mu+j\left(\mu_{C_{n}}+\mu_{Q}\right)}\right]^{-1}
$$

Simplifying,

$$
P_{L}=\frac{\frac{\left.\phi \rho^{(i-} c_{n}\right)}{\left(i-c_{n}\right) ! \prod_{j=1}^{i-c_{n}} c_{n} \mu+j\left(\mu c_{n}+\mu_{Q}\right)}}{\sum_{i=0}^{c_{n}} \frac{\phi \rho^{i}}{i !}+\sum_{i=c_{n+1}}^{\infty} \frac{\phi \rho^{i-c_{n}}}{\left(i-c_{n}\right) ! \prod_{j=1}^{i=c_{n}} c_{n} \mu+j\left(\mu_{C_{n}}+\mu_{Q}\right)}}
$$

In (9), $P_{L}$ is the Loss Probability, $C_{n}$ is the number of channels in cell, $i$ is the queue size and $\rho$ is the traffic intensity. The call completion rate (CCR) is given as

$C C R=\left(1-P_{L}\right) \times 100$

\section{PRESENTATION OF RESULTS}

The results of this study simulated in MATLAB are presented in the form of graphs.

\subsection{SYSTEM PARAMETERS}

System parameters and their values for the computation of the results are presented in this section. The maximum channel holding time was 2 minutes (120 seconds), traffic (in erlangs) was varied between the range of 1 and 100, maximum queuing time was 20 seconds, number of channels, varied from 1 to 32 and the signal power consideration $(\phi)$ was varied from 75 to 100 per cent. Details of the data are as presented in Table 1. 
Table 1: Table of parametric values for the scheme.

\begin{tabular}{ll}
\hline \multicolumn{1}{c}{ Parameter } & \multicolumn{1}{c}{ Value } \\
\hline Holding Time $\left(\mu_{C_{n}}\right)$ & 120 seconds \\
Queue Time $\left(\mu_{Q}\right)$ & 20 seconds \\
Traffic Intensity $(\rho)$ & Varied from 1 to 10 \\
Queue Size $(I)$ & Varied from 10 to 1000 \\
Power Consideration $(\phi)$ & Varied from $75-100 \%$ \\
Channel Size $\left(C_{n}\right)$ & Varied from 1 to 32 \\
\hline
\end{tabular}

Figure 3 is a graph showing the relationship between queue size and call completion rate. Figure 4 shows the effect of queue size on call completion at different traffic loads of 30, 50, 70 and 100 erlangs. Figure 5 depicts the impact of different queue sizes of 100, 500 and 1000 on call completion as traffic load increases.

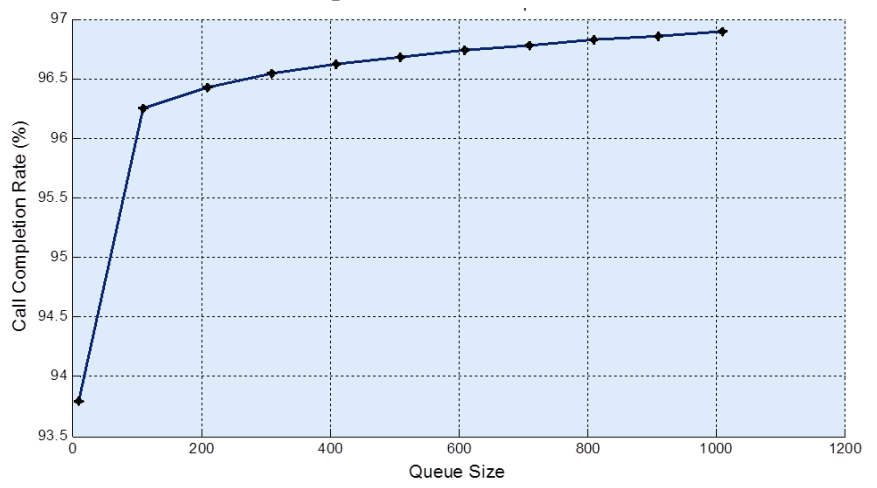

Figure 3: Queue size versus call completion rate.

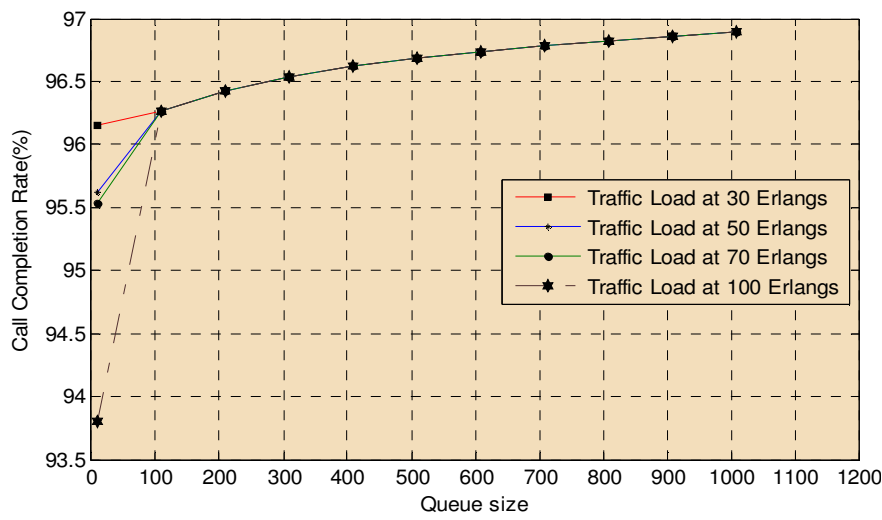

Figure 4: Call Completion Rate versus Queue size at different traffic loads.

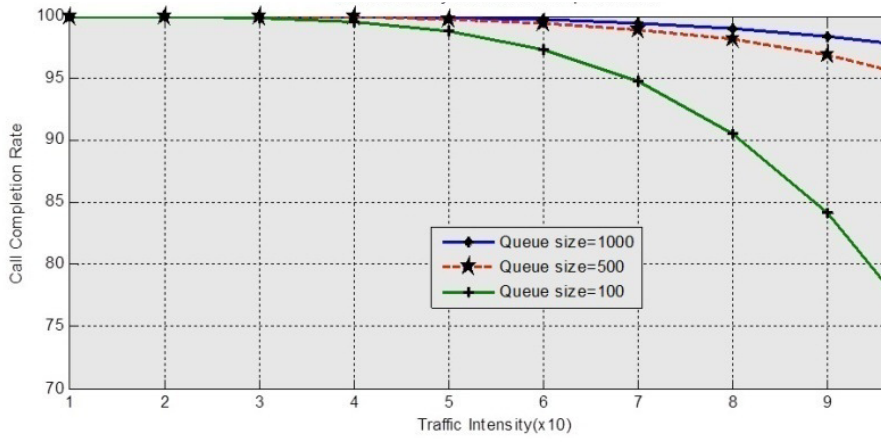

Figure 5: Call Completion rate versus traffic intensity at different queue sizes.

\section{DISCUSSION OF RESULTS}

The Figure 3 shows a sharp increase in the CCR for queue sizes 1 to 100 . However, above the queue size of 100, an increase in the CCR becomes gradual and smaller. This implies that the system does not require a very large queue, since the queue is regulated by a timer which clears all requests at the expiration of their set time. Figure 4 shows that between a queue size of 1 and 100 there is about 3\% increase in the CCR. The CCR for a traffic load of 50 Erlangs is higher than call completion rates at 70 and 100 Erlangs. Hence, the CCR increases with a decrease in the values of the traffic loads to 50 Erlangs. However, as the queue size increases, beyond 100 , the call completion rates at the different traffic loads converge, rising steadily (approximately 0.15\%) as queue size increases. This entails that a high queue size increases the probability that a call will be serviced even at high traffic loads. In figure 5 the queue reduces the effect of increasing traffic load. At the different queue sizes, the effect of increasing traffic load is the same. However, as traffic load exceeds 30 Erlangs, there is a divergence in CCR values at the different queue sizes, with the larger queue sizes having the better performances. Therefore, the larger the queue size, the better the performance of the network at high traffic loads.

\section{CONCLUSION}

The impact of queuing on call completion was investigated using a model which adopted a nonprioritised approach with blocked requests queued and serviced on a first-in first-out basis. The results in this paper show that queuing can minimise or may even eliminate the negative effect of high traffic on call completion rates.

\section{REFERENCES}

[1] Fang, Yuguang. "Performance Evaluation of wireless cellular networks under more realistic assumptions." Journal of Wireless Communication and Mobile Computing, 2005: 867-885.

[2] Georgeta Budura, Cornel Balint, Adrian Budura, Eugen Marza. "Traffic Models and Associated Parameters in GSM/(E) GPRS Networks." WSEAS transactions on communications 8, no. 8 (August 2009): 832-842.

[3] Akut.E.K, M.Sani. "Identification and Modelling of Dominant Causes of Congestion In Cellular Networks in Nigeria." IOSR Journal of Electronics and Communication Engineering (IOSR-JECE) 3, no. 2 (Sept- Oct 2012): 6-13. 
[4] Osahenvemwen O.A., Edeko F.O., Emagbetere J. "Traffic Modelling in Mobile Communication Networks." International Journal of Computer Applications (0975 - 8887), Volume 51- No.9, August 2012.

[5] M. Rajaratnam and F. Takawira, "Nonclassical traffic modeling and performance analysis of cellular mobile networks with and without channel reservation", IEEE Trans. Vehicular Technology, vol. 49, no. 3, May 2000, pp. 817-834.

[6] Z. Yan and S. Boon-Hee, "Handoff counting in hierarchical cellular system with overflow scheme", Computer Networks journal, Vol. 46, 2004, pp. 541554.

[7] R. Rodriguez-Dagnino and H. Takagi, "Counting handovers in a cellular mobile communication network: equilibrium renewal process approach", Performance Evaluation Journal, No. 52, April 2003, pp. 153-174.

[8] A. Zaim, H. Perros, and G. Rouskas, "Computing callblocking probabilities in LEO satellite constellations", IEEE Transactions on Vehicular Technology, vol. 52, pp. 622-636, May 2003.

[9] P. Fitzpatrick, M. Ivanovich, and J. Yin, "Models for pre-emption of packet data by voice in slotted cellular radio networks", Proceedings of IEEE Global Telecommunications Conference, vol. 2, pp. 16981702, November 2002.

[10] M. Alwakeel, "The Effect of Channel Holding Time Distribution on Handoff Failure Probability and Call Termination Probability" Proceedings of the World Congress on Engineering and Computer Science, Vol. II, pp.978-988,October 19-21, 2011.

[11] K. Yeo and C. Jun, "Teletraffic analysis of cellular communication systems with general mobility based on hyper-Erlang characterization", Journal of Computer and Industrial Engineering, vol. 42, pp. 507-520, 2002.
[12] Adegoke A.S and I.T Babalola. (2011). Quality of Service analysis for GSM telephone System in Nigeria. American Journal of scientific and Industrial Research, 710-712.

[13] Ayeni, A, "Quality of service profile for Nigerian GSM Networks" Journal of Engineering Research,University of Illorin, Department of Electrical Electronics,vol.1,2008.

[14] George P. Efthymoglou, Suwat Pattaramalai and Valentine A. Aalo, "Call Completion Probability with Generalized Call holding time and cell dwell time", IEEE, Bangkok, Thonburi, Thailand,2009.

[15] Toshihisa Ozawa, Nariaki Takahashi and Yukio Takahashi, "Bounds for Call Completion Probabilities in Large-Scale Mobile communications Networks". Journal of the Operations Research Society of Japan. Tokyo, Japan,2008.

[16] Mmekeme Nkop,Kufre Udofia,Constance Kalu, and Uduak Idio" Analysis of Call Completion Using the Time Diagram" International Journal of Engineering Research and Technology,vol.3,Issue 2,2014.

[17] Rappaport, Theodore S. Wireless Communication: Principles and Practice. Upper Saddle River, New Jersey: Prentice Hall PTR, 2002.

[18] School of Mechanical, Manufacturing and Medical Engineering. "MEN 170." System Modelling and Simulation. QUT, 2009.

[19] rfwireless. GSM Tutorials 2012. www.rfwirelessworld.com (accessed 04 12, 2013).

[20] Chadrasekaran, Balakrishnan. "Survey of Network traffic models." 2011. http://www.cse.wustl.edu (accessed 03 07, 2013).

[21] Cisco Systems. Traffic Analysis. 2010. http://www.cisco.com (accessed 04 12, 2013). 